\title{
Pregnancy and breast cancer
}

\author{
R Sasidharan MBBS FRACP and V Harvey MD FRACP \\ Department of Medical Oncology, Auckland City Hospital, Auckland, New Zealand
}

\begin{abstract}
Summary: Breast cancer is one of the most commonly diagnosed malignancies during pregnancy. Pregnancy-associated breast cancer $(\mathrm{PABC})$ presents a challenging clinical situation. This article reviews the current evidence around the management of $\mathrm{PABC}$ and the safety of pregnancy after breast cancer. The trend towards later age at first childbirth has resulted in an increase in the number of breast cancer cases coexistent with pregnancy. The management of breast cancer during pregnancy requires a multidisciplinary team approach. Breast surgery can be safely performed during any trimester of pregnancy. Radiation therapy, if required, must be delayed until after delivery. The majority of patients with PABC require chemotherapy. The timing of delivery in relation to chemotherapy administration should be carefully considered. There is no evidence to date that pregnancy termination influences overall survival for the mother. To date, there is no clear evidence that subsequent pregnancy after breast cancer is associated with worse maternal survival. There is a suggestion that subsequent pregnancy may in fact be associated with an improved survival. However, the available studies are limited by potential biases.
\end{abstract}

Keywords: pregnancy, breast cancer, chemotherapy, radiotherapy, prognosis

\section{INTRODUCTION}

Breast cancer is the most common malignancy and the second leading cause of cancer death in women. The relationship between pregnancy and breast cancer is complex. Pregnancy-associated breast cancer $(\mathrm{PABC})$ is defined as breast cancer diagnosed during pregnancy, in the first postpartum year or any time during lactation. Pregnancy complicates the management of breast cancer as treatment needs to balance survival of the mother against the potential risks to the fetus. There is a paucity of randomized data in this field. The majority of the information comes from retrospective case reviews, single institution series and case reports.

\section{INCIDENCE}

Breast cancer is the most common solid tumour in pregnancy, after cervical carcinoma, ${ }^{1}$ with an incidence of one in 3000 pregnancies. ${ }^{2}$ It has been estimated that up to $3 \%$ of all breast cancers are diagnosed in pregnant or lactating women. ${ }^{3}$ With the increasing trend for women to delay child-bearing, the incidence of PABC is expected to increase. Evidence from the Surveillance Epidemiology and End Results cancer statistics data indicate that women who have their first pregnancy before the age of 20 years have a 2-3 times lower risk of developing breast cancer than women who have their first pregnancy after the age of 30 years. ${ }^{4}$ Pregnancy transiently increases the risk of developing breast cancer, which is most pronounced in women who are older at the time of their first childbirth. This is thought to be due to pregnancy stimulating the growth of

Correspondence to: Rita Sasidharan

Email: ritas@adhb.govt.nz cells which have already undergone the early stages of malignant transformation. However, previous pregnancy has a protective effect on subsequent postmenopausal breast cancer by inducing the differentiation of normal mammary stem cells that have the potential for neoplastic change. ${ }^{5,6}$ Although the available data are limited, there is a suggestion that women with a genetic predisposition to breast cancer may be overrepresented among pregnant women with cancer. ${ }^{7}$

\section{DIAGNOSIS AND STAGING}

Delay in diagnosis is common in breast cancer occurring during pregnancy or lactation. This is partly due to the physiological changes in the breast during pregnancy and lactation, which may mask the changes from cancer. Moreover, women of childbearing age are not undergoing regular mammographic screening. This underscores the importance of undertaking a full physical examination, including breast examination, at the first and subsequent prenatal visits of every pregnant woman. According to a Norwegian study, the estimated delay in breast cancer diagnosis due to pregnancy is 2.5 months and due to lactation is six months. ${ }^{8}$ It is generally thought that a pregnant woman has a 2.5-fold higher risk of presenting with advanced breast cancer than non-pregnant women. ${ }^{2}$

Imaging modalities used for diagnosis and staging of breast cancer in pregnant women need to be modified to avoid or minimize radiation exposure to the fetus. Mammography is not as useful in pregnant patients as in non-pregnant women. The sensitivity is lessened due to increased density in the breast parenchyma associated with pregnancy. The concern regarding fetal radiation exposure from mammography is probably overstated. Approximate fetal radiation load is 4 mGy from mammography, which is considered low risk. ${ }^{9}$ 
However, ultrasonography is the imaging modality of choice for clarifying an abnormality and in distinguishing between a solid and cystic lesion. Magnetic resonance imaging (MRI) with gadolinium contrast agents is generally avoided in the first trimester but may be used later in pregnancy if indicated. Definitive diagnosis is made by core biopsy. Risk of falsepositive or false-negative diagnosis is higher with fine needle aspiration in PABC, due to the hyperproliferative cellularity of the breast tissue during pregnancy and lactation. For this reason, it is imperative that the pathologist be informed that the woman is pregnant or lactating. ${ }^{10}$

After a diagnosis is made, the extent of disease is evaluated. As with non-pregnant patients with breast cancer, the TNM staging system of the American Joint Committee on Cancer is used to describe the extent of breast cancer in PABC. The safety and efficacy of sentinel lymph node biopsy is not fully evaluated in the setting of PABC. The common sites of metastatic spread are bone, liver and lungs. Bone scans are not routinely recommended for bone evaluation in pregnancy because of the associated radiation risk to the fetus. MRI of the skeleton may be used. Chest radiography with abdominal shielding and abdominal ultrasounds are safe procedures for pregnant women for evaluation of lung and liver metastasis, respectively. Computed tomography should be avoided in pregnancy because of the potential radiation dose. ${ }^{11}$

\section{PATHOLOGY}

Breast cancers occurring during pregnancy are usually higher grade infiltrating ductal carcinomas. ${ }^{12}$ They are often associated with larger tumour size and higher nodal burden. There is unresolved controversy as to whether this is due to delay in diagnosis or whether the pregnancy-associated altered vascularity, increased serum hormone levels and immunosuppression result in acceleration of tumour dynamics. ${ }^{10}$ Oestrogen receptors and progesterone receptors are frequently negative in PABC as shown in Table 1. The high circulating concentrations of steroids in pregnancy might interfere with the assays used in determining hormonal receptor status. ${ }^{13}$ Whether there is a higher incidence of HER2 expression in pregnant women compared with non-pregnant women is unclear from the existing studies. Between $28 \%$ and $58 \%$ of PABC are reported to be HER2 positive. There has been much debate as to whether these poor prognostic features are specific features of PABC or whether they simply reflect the biology associated with breast cancer occurring in this younger age group.

\section{MANAGEMENT}

Breast cancer management in pregnancy requires a multidisciplinary team approach. There is no evidence, to date, that pregnancy termination improves outcome in PABC. ${ }^{19}$ Generally, overall management of breast cancer in a pregnant woman follows the same guidelines as their non-pregnant counterpart and depends on the stage of the cancer. However, timing of therapy may have to be adapted to take into account fetal risks.

\section{LOCO-REGIONAL TREATMENT}

Surgery is the definitive primary treatment for women with early breast cancer whether diagnosed during pregnancy or not. Exceptions are inflammatory and locally advanced breast carcinomas where primary medical treatment (neoadjuvant therapy) is recommended prior to surgery. Traditionally mastectomy with axillary node dissection has been the standard in PABC. However, similar survival rates have been reported with breast-conserving surgery and mastectomy in pregnant women. ${ }^{20}$ While some investigators consider sentinel lymph node biopsy to be safe in PABC, its safety and efficacy has not been fully evaluated and it should not be used as a standard practice until more data are available. Additionally, there is a high incidence of axillary lymph node metastases in PABC and hence axillary lymph node dissection is regarded as the gold standard.

Local radiotherapy is usually indicated for the majority of patients with PABC because of the advanced tumour stage. Radiotherapy of the breast and/or chest wall should be delayed until after delivery, because of the risk of high-dose radiation to the fetus.

\section{SYSTEMIC THERAPY}

Neoadjuvant or adjuvant chemotherapy is required for the vast majority of women with PABC. Careful consideration should be given in the selection of chemotherapeutic agents and the timing of chemotherapy in pregnant women. The benefit to the mother must be balanced against the risk to the fetus. As a general rule, all chemotherapeutic agents should be avoided during the first trimester, when organogenesis is not complete. There are significantly higher rates of miscarriage and fetal malformation when chemotherapy is administered during the first trimester. The incidence of fetal malformations is reported

Table 1 Biological features of pregnancy-associated breast cancer

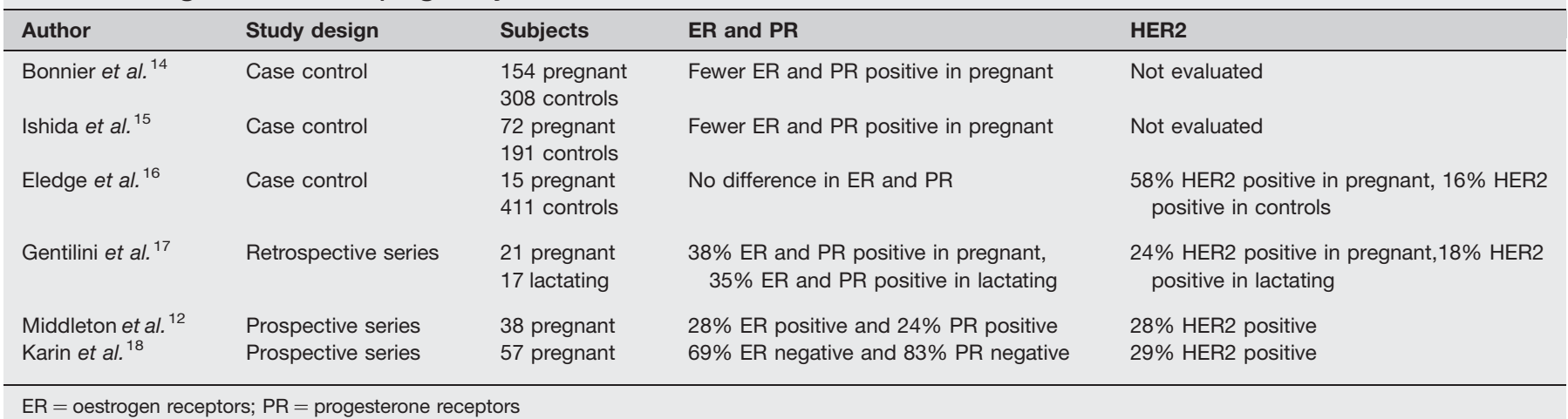

$\mathrm{ER}=$ oestrogen receptors; $\mathrm{PR}=$ progesterone receptors 
to be around $16 \%$ with chemotherapy use in the first trimester compared with an incidence of $1.3 \%$ thereafter. $^{21}$

Chemotherapy has been much more widely used in second and third trimesters. The most common adverse effects reported with such use in the second and third trimesters are intrauterine growth retardation, premature labour and low birth weight. ${ }^{22}$ A single institution series reported the maternal and fetal outcomes of 22 patients with PABC. ${ }^{23}$ Seventeen patients were diagnosed during pregnancy and five were diagnosed during lactation. Chemotherapy was initiated during the 10 th week in four cases diagnosed during the first trimester. No congenital malformation was reported in these children exposed to chemotherapy during the late first trimester. Seven patients were treated with a combination of doxorubicin, flurouracil and cyclophosphamide and four were treated with docetaxel during the second and third trimesters. There were no reports of congenital malformation. At a median follow-up of 40 months, 17 patients were alive and disease free, and five had died of metastatic breast cancer.

In another series from five London teaching hospitals, none of the 27 children who were exposed to chemotherapy in utero showed congenital malformation. ${ }^{24}$ Sixteen of these children were exposed to anthracycline-based therapy and 11 were exposed to CMF chemotherapy (cyclophosphamide, methotrexate, fluorouracil). One of these patients was treated during the first trimester with CMF chemotherapy and a spontaneous abortion resulted. Disease-free survival was $63 \%$ at 40-month follow-up.

The largest prospective series is from MD Anderson Cancer Center. ${ }^{18}$ In this study, 57 pregnant women with localized invasive breast cancer were treated with fluorouracil, adriamycin, cyclophosphamide (FAC) chemotherapy on a single arm, Institutional Review Board approved the protocol. Thirty-two patients received adjuvant chemotherapy and 25 received neoadjuvant chemotherapy. The median gestational age at the time of chemotherapy initiation was 23 weeks, range 11-34 weeks. Despite presenting with more advanced stage, $70 \%$ were alive and disease free at 38.5 months follow-up. One woman died due to pulmonary embolism following a caesarean section. The median gestational age at delivery was 37 weeks and median birth weight was $2890 \mathrm{~g}$. There were no stillbirths, perinatal deaths or miscarriages. Neonatal complications were comparable to the reported incidence among general population. Ninety-seven percent of the offspring had normal development when compared with siblings or other children in the family. The authors concluded that breast cancer can be treated with FAC chemotherapy during the second and third trimesters without significant complications.

Anthracyclines are among the most effective drugs in breast cancer and the greatest experience during pregnancy has been reported for doxorubicin. Cyclophosphamide, an alkylating agent and 5-fluorouracil (antimetabolite) are an integral part of many common chemotherapy regimens in the treatment of breast cancer. They appear to be relatively safe from the second trimester. Limited safety data are available for the use of taxanes. Although there is no reported case of congenital malformation, sequential taxane therapy after delivery is the common practice. The safety of Trastuzumab (Herceptin) during pregnancy is not fully evaluated. Reversible oligohydramnios/anhydramnios have been reported in a few cases. ${ }^{25}$

In all the published series, the median follow-up of children exposed to chemotherapy in utero is relatively short, hence the long-term effect of treatment is uncertain. The potential long-term effects may include infertility, neurological impairment, risk of future malignancies and increased risk of damage to the heart, lung or kidneys. However, there are limited data on which to base a conclusive opinion.

\section{ENDOCRINE THERAPY}

Endocrine therapy with selective oestrogen receptor modulators such as tamoxifen is contraindicated during pregnancy. It has been associated with spontaneous abortions and fetal malformations including craniofacial malformations and ambiguous genitalia. ${ }^{26}$ Whether in utero exposure to tamoxifen increases the incidence of gynaecological cancers later on is unknown. The recommended practice is to defer endocrine therapy until after delivery.

\section{CONTRACEPTION AFTER BREAST CANCER}

Premenopausal women with breast cancer should be advised regarding contraception during the period of their treatment and afterwards. For women who have completed their families, a permanent method of contraception such as sterilization could be considered. For women who have not completed their families or who do not wish to consider a permanent method, intrauterine contraception or barrier contraception are options. Hormonal contraceptives are contra-indicated in women with a hormone receptor-positive breast cancer and may be deleterious even in those with hormone receptornegative disease given the high incidence of relapse in trials of hormone replacement therapy in this group. ${ }^{27,28}$ Gonadotropin releasing hormone (GnRH) analogues, which are used either in the adjuvant setting for temporary ovarian suppression in hormone receptor-positive cancers or along with chemotherapy for their possible benefit in preventing ovarian toxicity of chemotherapy will also serve the purpose of contraception. However, this is not recommended during chemotherapy in women with hormone receptor-positive cancers because of the possibility that GnRH might reduce the efficacy of concomitant chemotherapy. ${ }^{29}$

\section{PREGNANCY AFTER BREAST CANCER}

The issue of pregnancy following breast cancer treatment is a complex issue for breast cancer survivors. It is impossible to conduct a randomized controlled trial on this subject, so the evidence upon which guidelines and recommendations are based comes from retrospective analysis and population-based studies. Early diagnosis and more effective treatment have resulted in higher event-free and overall survival for women with breast cancer. Given the rising mean age at first pregnancy in the general population, there are an increasing number of women with breast cancer diagnosis who have not completed their families. These women, and others with breast cancer, require detailed preconception counselling including presentation of all relevant information from the literature to enable them to make an informed decision with regard to a further pregnancy after the diagnosis is made.

Oestrogens play a significant role in breast carcinogenesis. Increase in risk of breast cancer recurrence due to high levels of gestational oestrogen and progesterone has been a theoretical 
concern when advising women regarding subsequent pregnancy. Breast cancer survivors in the past were advised to avoid pregnancy. Although to date there is no evidence that pregnancy after the diagnosis of breast cancer influences maternal survival, counselling must take into account the longterm prognosis and its impact on the woman and her family.

Kroman et al. ${ }^{30}$ investigated the prognostic influence of pregnancy after breast cancer treatment based on a linkage analysis between the population-based Danish Breast Cancer Cooperative Group registry and other national registries. In their 10-year update ${ }^{31}$ of the study, among the 10,236 women with a primary breast cancer aged 45 years or less, 371 women experienced pregnancy after treatment of breast cancer. Multivariate analysis showed that women who had a full-term pregnancy subsequent to breast cancer therapy had a reduced risk of dying (risk ratio [RR]: 0.73, 95\% confidence interval [CI]: $0.54-0.99$ ). The effect was not modified by age at diagnosis, tumour size, nodal status or reproductive history before the diagnosis of breast cancer. The International Breast Cancer Study Group (IBCSG) conducted a retrospective review of survival for women who became pregnant after treatment for early-stage breast cancer in institutions associated with IBCSG in comparison with that of matched patients selected from among IBCSG trial participants. ${ }^{32}$ The mean interval $( \pm S D)$ from diagnosis of breast cancer to completion of first subsequent pregnancy was $33.0 \pm 21.1$ months (median 27.8 months). Overall five- and 10-year survival measured from the diagnosis of early-stage breast cancer among the 94 study group patients were $92 \pm 3 \%$ and $86 \pm 4 \%$, respectively, compared with $85 \pm 3 \%$ at five years and $74 \pm 4 \%$ at 10 years for the matched comparison group (RR: $0.44,95 \%$ CI: $0.21-0.96$; $P=0.04)$.

A population-based study from Western Australia by Ives et al. ${ }^{33}$ is also reassuring. This study identified 2539 women aged 15-44 with a breast cancer diagnosis between 1982 and 2000. Of these women, $123(5 \%)$ had at least one pregnancy after their diagnosis and before 31 December 2004. Median time from diagnosis to first subsequent pregnancy was 23 months. Fifty percent of women conceived within two years of diagnosis. The five-year overall survival was $92 \%$ and 10 -year overall survival was $86 \%$. Subsequent pregnancy was associated with an improved overall survival in those women who waited 24 months to become pregnant (hazard ratio: 0.48 ; 95\% CI: $0.27-$ $0.83 ; P=0.009)$. Survival was the same in those women who waited at least six months to become pregnant.

There have been several reviews of the literature regarding pregnancy following a diagnosis of breast cancer. Upponi et al. ${ }^{34}$ concluded that based on available data, the effect of a subsequent pregnancy on patients who have had breast cancer with regard to local recurrence, distant metastasis and survival remains uncertain and difficult to quantify for individual patients. Chabbert-Buffet et al. ${ }^{35}$ reported that pregnancy should be possible for most breast cancer survivors, provided that the initial prognosis was good. Interpretation of data from population-based and retrospective studies is problematic. Women who become pregnant after breast cancer are a highly selected group. The trend to a survival benefit with subsequent pregnancy seen in these studies may merely represent a 'healthy mother effect'. Whether there is an unexplained biological beneficial effect with subsequent pregnancy on prognosis is uncertain, but the fact that there is an approximate $15 \%$ ten-year mortality for this group of women should not be overlooked and its potential impact on a woman's family should be discussed in preconception counselling. Conventional advice is to wait two years before conception after breast cancer diagnosis to get through the period of early recurrence risk.

\section{CONCLUSIONS}

The incidence of PABC is increasing. While the types of investigation need to be tailored and the timing of treatment must take the gestational age of the fetus into account, loco-regional treatment and adjuvant chemotherapy can generally follow the management guidelines as in a non-pregnant woman. Endocrine therapy and radiation therapy, if indicated, are given only after delivery.

The issues around pregnancy after breast cancer diagnosis are complex. Subsequent pregnancy should be discussed carefully, reflecting all the current data and stressing the uncertainties where they exist. The timing of a subsequent pregnancy should be based on individual circumstances, the requirement for adjuvant endocrine therapy and the prognosis of the tumour.

Conflict of interest statement: The authors indicated no potential conflicts of interest.

\section{REFERENCES}

1 Antonelli NM, Dotters DJ, Katz VL, Kuller JA. Cancer in pregnancy: a review of the literature. Obstet Gynecol Survey 1996;51:125-34

2 Pavilidis N, Penthroudakis G. The pregnant mother with breast cancer: diagnostic and therapeutic management. Cancer Treat Rev 2005;31:439-47

3 Ring AE, Smith IE, Ellis PA. Breast Cancer and Pregnancy. Ann Oncol 2005; 16:1855-60

4 Ries LAG, Eisne NP, Kosary CL, et al., eds. SEER Cancer Statistics Review 1973-1999. Bethseda, MD: National Cancer Institute, 2002. Available from http://seer.cancer.gov/csr

5 Lambe M, Itseich C, Trichopoulos D, et al. Transient increase in the risk of breast cancer after giving birth. N Engl J Med 1994;331:5-9

6 Ann P. Pregnancy and breast cancer. Oncology 2005;19:1-6

7 Johannsson O, Loman N, Borg A, Olsson H. Pregnancy-associated breast cancer in BRCA1 and BRCA2 germline mutation carriers. Lancet 1998;352: 1359-60

8 Tretli S, Kvalheim G, Thoresen S, Host H. Survival of breast cancer patients diagnosed during pregnancy or lactation. Br J Cancer 1988;58:382-4

9 Nicklas AH, Baker ME. Imaging strategies in the pregnant cancer patient. Semin Oncol 2000;27:623-32

10 Janni W, Hepp P, Nestle-Kraeming C, et al. Treatment of pregnancy-associated breast cancer. Expert Opin Pharmacother 2009;10:2259-67

11 Lobil S, von Minckwitz G, Gwyn K, et al. Breast carcinoma during pregnancy. International recommendations from an expert meeting. Cancer 2006;106: 237-46

12 Middleton LP, Amin M, Gwyn K, et al. Breast carcinoma in pregnant women assessment of clinicopathologic and immunohistochemical features. Cancer 2003;98:1055-60

13 Navrozoglou I, Vrekoussis T, Kontostolis E, et al. Breast cancer during pregnancy: A mini review. Eur J Surg Oncol 2008;34:837-43

14 Bonnier P, Romain S, Dilhuydy JM, et al. Influence of pregnancy on the outcome of breast cancer: a case control study. Int J Cancer 1997;72:720-7

15 Ishida T, Yokoe T, Kasumi F, et al. Clinicopathologic characteristics and prognosis of breast cancer patients associated with pregnancy and lactation: analysis of case-control study in Japan. Jpn J Cancer Res 1992;83:1143-9

16 Eledge RM, Ciocca DR, Langone G, et al. Estrogen receptor, progesterone receptor and Her-2/neu protein in breast cancer from pregnant patients. Cancer 1993;71:2499-506

17 Gentilini O, Masallo M, Rotmensz N, et al. Breast cancer diagnosed during pregnancy and lactation: biological features and treatment options. Eur J Surg Oncol 2005;31:232-6

18 Karin M, Hahn E, Johnson PH, et al. Treatment of pregnant breast cancer patients and outcomes of children exposed to chemotherapy in utero. Cancer 2006;107:1219-26 
19 King R, Welch J, Martin J, et al. Carcinoma of the breast associated with pregnancy. Surg Gynecol Obstet 1985;160:228-32

20 Kuerer H, Ames GK, et al. Conservative surgery and chemotherapy for breast carcinoma during pregnancy. Surgery 2002;131:108-10

21 Doll DC, Ringenberg QS, Yabro JW. Antineoplastic agents and pregnancy. Semin Oncol 1989;16:337-46

22 Zemlickis D, Lishner M, Degendorfer P, et al. Fetal outcome after in utero exposure to cancer chemotherapy. Arch Intern Med 1992;152:573-6

23 Garcia-Manero M, Rogo MP, Espinos J, et al. Pregnancy associated breast cancer. Eur J Surg Oncol 2009;35:215-8

24 Ring AE, Smith IE, Jones A, et al. Chemotherapy for breast cancer during pregnancy, an 18-year experience from five London Teaching Hospitals. J Clin Oncol 2005;23:4192-7

25 Badder AA, Schlembach D, Tarnussino KF, et al. Anhydramnios associated with administration of Trastuzumab and Paclitaxel for metastatic breast cancer during pregnancy. Lancet Oncol 2007;8:79-81

26 Issacs RJ, Hunter W, Clark K. Tamoxifen for systemic treatment of advanced breast cancer during pregnancy - case report and literature review. Gynecol Oncol 2001;80:405-8

27 Holmberg L, Iverson O-E, Magnus C, et al. Increased risk of recurrence after hormone replacement therapy in breast cancer survivors. J Natl Cancer Inst 2008;100:475-82
28 Pritchard KI. Should observational studies be a thing of the past? J Natl Cancer Inst 2008;100:451

29 Emons G, Grundker C, Gunthert AR, et al. GnRH agonists in the treatment of Gynaecological and breast cancers. Endocrine Relat Cancer 2003;10:291-9

30 Kroman N, Jensen M-B, Melbye M, et al. Should women be advised against pregnancy after breast cancer treatment? Lancet 1997;350:319-22

31 Kroman N, Jensen MB, Wohlfahrt J, et al. Pregnancy after treatment of breast cancer - a population based study on behalf of Danish Breast Cancer Cooperative group. Acta Oncol 2008;47:545-9

32 Gelber S, Coates AS, Goldhirsch A, et al. Effect of pregnancy on overall survival after the diagnosis of early stage breast cancer. J Clin Oncol 2001;19:1671-5

33 Ives A, Saunders C, Bulsara M, et al. Pregnancy after breast cancer: population based study. BMJ 2007;334:194

34 Upponi SS, Ahmad F, Whitaker IS, Purushotham AD. Pregnancy after breast cancer. Eur J Cancer 2003;39:736-41

35 Chabbert-Buffet N, Uzan C, Gligorov J, et al. Pregnancy after breast cancer: A need for global patient care, starting before adjuvant therapy. Surg Oncol 2009;19:47-55, doi:10.1016/j.suronc.2009.03.009

(Accepted 23 March 2010) 